\title{
Structural Basis for Binding of Cyclic 2-Oxoglutarate Analogues to Factor-Inhibiting Hypoxia-Inducible Factor
}

\author{
Ana Conejo-Garcia ${ }^{\mathrm{a}}$, Michael A. McDonough**a ${ }^{\mathrm{a}}$ Christoph Loenarz ${ }^{\mathrm{a}}$, Luke A. McNeill ${ }^{\mathrm{a}}$, \\ Kirsty S. Hewitson ${ }^{\mathrm{a}}$, Wei Ge ${ }^{\mathrm{a}}$, Benoît M. Liénard ${ }^{\mathrm{a}}$, Christopher J. Schofield ${ }^{\mathrm{a}}$ and Ian J. \\ Clifton* ${ }^{\mathrm{a}}$
}

${ }^{a}$ The Department of Chemistry, Chemistry Research Laboratory, University of Oxford, 12 Mansfield Road, Oxford, OX1 3TA, United Kingdom.

*Correspondence should be addressed to I.J.C. (ian.clifton@chem.ox.ac.uk) or M.A.M. (michael.mcdonough@chem.ox.ac.uk)

\begin{abstract}
Aromatic analogues of the 2-oxoglutarate cosubstrate of the hypoxia-inducible factor hydroxylases are shown to bind at the active site iron: Pyridine-2,4-dicarboxylate binds as anticipated with a single molecule chelating the iron in a bidentate manner. The binding mode of a hydroxamic acid analogue, at least in the crystalline state, is unusual because two molecules of the inhibitor are observed at the active site and partial displacement of the iron binding aspartyl residue was observed.
\end{abstract}

Keywords: factor inhibiting hypoxia-inducible factor; oxygenase; hypoxic response; hydroxamic acid; 2-oxoglutarate analogue

2-Oxoglutarate (2OG) and ferrous iron dependent oxygenases (2OG oxygenases) play important roles in regulating the hypoxic response in humans via the post-translational hydroxylation of hypoxia-inducible factor. Prolyl- and asparaginyl- hydroxylation of the HIF$\alpha$ subunit signals for their degradation and blocks the interaction of HIF- $\alpha$ with transcriptional co-activators, respectively. In humans there are three HIF prolyl hydroxylase isoforms (PHD1,2,3) and one HIF asparaginyl hydroxylase (FIH, factor-inhibiting HIF) (for reviews see $\left.{ }^{1-3}\right)$. $2 \mathrm{OG}$ oxygenase homologues of FIH have been shown to catalyse the demethylation of $N^{\varepsilon}$-methylated lysine-residues in histones (for review see ${ }^{4}$ ). Because of their roles in the hypoxic response and chromatin regulation, human $20 \mathrm{O}$ oxygenases are current pharmaceutical targets.

There is interest in small molecules that target specific human $20 \mathrm{O}$ oxygenases both for therapeutic and functional assignment purposes. Most reported 2OG oxygenase inhibitors are either $\mathrm{Fe}$ (II) chelators and/or $2 \mathrm{OG}$ analogues. Relatively few structures of human $2 \mathrm{OG}$ oxygenases with these inhibitors are available. Structures for FIH and the histone demethylase JMJD2A in complex with $\mathrm{N}$-oxalyl amino acid 2OG analogues, including $\mathrm{N}$ oxalyl glycine and $N$-oxalyl-D-phenylalanine have been described (PDB-ID 1H2K, 1H2M, and 1YCI). ${ }^{5,6}$ However, few structures of human $2 \mathrm{OG}$ oxygenases in complex with conformationally constrained analogues of 2OG have been reported (PDB-ID 2G1M, 2G19, $2 \mathrm{G} 1 \mathrm{M}){ }^{7,8}$ Because of their different roles in the hypoxic response, the identification of inhibitors that are selective for FIH or the PHDs is of interest. The development of selective $\mathrm{FIH}$ inhibitors may be challenging because $\mathrm{FIH}$, is closely related to the JmjC domain histone 
demethylase family, more so than are the PHDs. Here we report the synthesis of monocyclic $2 \mathrm{OG}$ analogue inhibitor templates and three crystal structures of these compounds in complex with FIH. The results should be useful for the design of more potent and selective FIH inhibitors.

Pyridine-2,4-dicarboxylate 4 and 3-hydroxy pyridine-2-carbonyl glycine $\mathbf{6}$ are inhibitors of the pro-collagen and HIF prolyl hydroxylases. ${ }^{9-11}$ The other cyclic $2 \mathrm{OG}$ analogues tested were prepared by synthesis (Figure 1). Compound $\mathbf{3}$ was prepared from ethyl 3hydroxybenzoate as reported. ${ }^{12}$ (3-Hydroxyphenyl)(oxo)acetic acid $\mathbf{2}$ was prepared via a route involving base mediated rearrangement of a cyanohydrin carbonate ester (Scheme 1). Aromatic cyanohydrin ester (9) was prepared by the method of Thasana et al. ${ }^{13}$, employing ethyl chloroformate, aqueous $\mathrm{NaCN}$ and a phase transfer catalyst. We found that employing THF rather than $\mathrm{CH}_{2} \mathrm{Cl}_{2}$ as the organic phase resulted in higher yields and avoided the need for purification by distillation. Thiol $\mathbf{5}$ was prepared from 3-hydroxyphenyl acetic acid in seven steps (Scheme 2), in a route including benzylic bromination. The basic deprotection conditions led to isolation of the disulfide of the target thiol 5. The free thiol was generated by treatment with dithiothreitol immediately prior to assays.

The cyclic $2 \mathrm{OG}$ analogues were then evaluated for FIH inhibition using both $1-\left[{ }^{14} \mathrm{C}\right]-2 \mathrm{OG}$ turnover assay (monitoring ${ }^{14} \mathrm{CO}_{2}$ production) and MALDI MS and also as inhibitors of the catalytic domain of PHD2 using a homogeneous time-resolved fluorescence (HTRF) assay for comparison. ${ }^{14}$ None of the compounds tested were potent inhibitors. ${ }^{9-11}$ The IC $_{50}$ values were in the $\mathrm{mM}$ range under standard assay conditions $(2: 1 \mathrm{mM} ; \mathbf{3}: 18 \mu \mathrm{M} ; 4: 625 \mu \mathrm{M} ; 50.5$ $\mathrm{mM}$, 6: $91 \mu \mathrm{M}$; 6a: >1 mM). Similar results were observed with PHD2, with none of the compounds tested causing more than a 50\% drop in activity when assayed at $1 \mathrm{mM}$ concentration using the HTRF assay, with the exception that the reported PHD inhibitors ${ }^{9-11}$ pyridine-2,4-dicarboxylate 4 and 3-hydroxy pyridine-2-carbonyl glycine $\mathbf{6}$ were observed to inhibit more potently than the other cyclic inhibitors. The relative lack of potency of $\mathbf{4}$ with FIH compared to PHD2 is interesting given the conserved metal-coordination chemistry in $2 \mathrm{OG}$ oxygenases and prompted us to carry out structural investigations on $\mathrm{FIH}$. Crystal structures were obtained for compounds $\mathbf{2}, \mathbf{3}$ and $\mathbf{4}$ in complex with FIH.

The anticipated close relationship of 2 to the conformation of $2 \mathrm{OG}$ observed at the FIH active site (PDB-ID $1 \mathrm{H} 2 \mathrm{~L}, 1 \mathrm{H} 2 \mathrm{~N})^{15}$ is supported by the crystallographic analysis. 2 binds to the $\mathrm{Fe}(\mathrm{II})$ in a similar manner as $2 \mathrm{OG}$ with its carbonyl oxygen trans to His 199 and its ketone oxygen trans to Asp201 (Figures 2b and S1b). The sidechain carboxylate of $\mathbf{2}$ forms the same interactions as that of 2OG. The benzyl ring of $\mathbf{2}$ is located in a hydrophobic pocket sandwiched between Leu188, Ile281, Phe207, and $\mathrm{C}^{\varepsilon}$ of Lys214. This structure reveals that monocyclic $2 \mathrm{OG}$ analogues can bind at the active site in a manner closely related to $2 \mathrm{OG}$ and encouraged further work on other $2 \mathrm{OG}$ analogues.

As anticipated from structural work on the histone demethylase JMJD2A ${ }^{8}$, pyridine-2,4dicarboxylate 4 is coordinated to the FIH active site $\mathrm{Fe}(\mathrm{II})$ in a bidentate manner via its pyridinyl nitrogen and 2-carboxylate, with its $\mathrm{C} 4$ carboxylate interacting with the same residues as the $20 G \mathrm{C} 4$ carboxylate. Unanticipated electron density at the active site was modelled and refined as a glycerol molecule (the cryoprotectant used), which is positioned to coordinate to the $\mathrm{Fe}$ (II) and the side chain of one of the Fe(II) coordinating residues, Asp201 (Figures 2a and S1a). Comparison of the FIH.4 structure with other FIH structures reveals several differences. The pyridine ring of $\mathbf{4}$ is observed bound to FIH in the same plane as for $2 \mathrm{OG}$ and the analogues NOG, succinate, and fumarate (PDB-IDs 2CGO, 2CGN, 1H2M, 
$1 \mathrm{H} 2 \mathrm{~L}, 1 \mathrm{H} 2 \mathrm{~N}$, and $1 \mathrm{H} 2 \mathrm{~K}) .{ }^{15,16}$ However, in the case of the FIH structure in complex with 4, compared to the other structures there is an $\sim 80^{\circ}$ rotation of the $\mathrm{C} \beta-\mathrm{C} \gamma$ bond in His 199 , the side-chain of which coordinates the $\mathrm{Fe}(\mathrm{II})$, likely due to a steric interaction with the pyridine ring of 4 . The apparent inability of 4 to bind to the Fe(II) of FIH in an un-encumbered manner may rationalise its relative lack of potency.

Comparison of the binding modes of 4 with FIH and JMJD2A (PDB-ID 2VD7) reveals a striking difference, in that the orientations of the plane of the pyridine ring of $\mathbf{4}$ are different. Although 4 binds in the same plane as 2OG/NOG in FIH, it binds in a different plane in JMJD2A. This is likely due to the different steric constraints within the JMJD2 subfamily active site.

Because hydroxamic acids are clinically used as metallo-enzyme inhibitors, we then determined the structure of FIH in complex with 3. Interestingly, the electron density map implied that two molecules of $\mathbf{3}$ were bound to the $\mathrm{Fe}(\mathrm{II})$ at the active site. In one binding mode, $\mathbf{3}$ was observed to bind in a manner closely related to that of $2 \mathrm{OG}, \mathbf{2}$, and $\mathbf{4}$, with the hydroxamic acid observed to bind in the anticipated bidentate manner. The second binding mode was unexpected because it apparently involves the partial displacement of the Asp201 carboxylate from coordinating to the Fe(II).

The hydroxamate nitrogen of $\mathbf{3}$ is positioned to form a hydrogen bond with the Asp201 side chain oxygen stabilising its non-metal ligated position. The unexpected second binding mode of $\mathbf{3}$ with displacement of the Asp201 side chain from its coordination to $\mathrm{Fe}(\mathrm{II})$ may in part reflect the apparent unusual behaviour of FIH with respect to its metal cofactor. Previous structural and biochemical analyses demonstrated that substitution of Asp201 by glycine or alanine residues does not ablate Fe(II) binding and the D201G variant retains hydroxylation activity (PDB-IDs 3D8C and 2ILM). ${ }^{17}$

Overall the results demonstrate that cyclic 2OG analogues can bind at the FIH active site in a closely related manner to which its $2 \mathrm{OG}$ co-substrate binds. Appropriate structure guided functionalisation of these templates should enable the generation of more potent and selective inhibitors with precedence from work on the JMJD2 family of histone demethylases. ${ }^{8,}{ }^{18}$ The results also demonstrate that hydroxamic acids can bind to the active site transition metal centre in 2OG oxygenases in a similar manner to which they inhibit zinc dependent hydrolases. ${ }^{19}$ This observation is of interest because the clinically used hydroxamic acid containing histone decetylase inhibitor SAHA was reported to also inhibit the JMJD2 enzymes. ${ }^{18}$ Finally, the observation that two molecules of the hydroxamic acid $\mathbf{3}$ bind at the active site metal of FIH, apparently causing the aspartyl residue that normally coordinates to move away from the metal, suggests that it may be possible to identify inhibitors of $2 \mathrm{OG}$ oxygenases that work via novel mechanisms, such as by ejection of the active site iron.

Coordinates for FIH in complex with 2, 3, and $\mathbf{4}$ have been deposited in the PDB as 2WA3, $2 \mathrm{WA} 4$, and 2W0X, respectively. Further description of the structures and details of synthesis, assays, and crystallography are provided as Supplementary Information.

Acknowledgements: We thank the BBSRC, the Wellcome Trust, the European Commision : Marie Curie Programme MEIF-CT-2003-500525 (A.C-G) and the European Union for funding. 


\section{References}

1. Hirota, K.; Semenza, G. L. Biochem. Biophys. Res. Commun. 2005, 338, 610.

2. Kaelin, J. W. G. Biochem. Biophys. Res. Commun. 2005, 338, 627.

3. Schofield, C. J.; Ratcliffe, P. J. Biochem. Biophys. Res. Commun. 2005, 338, 617.

4. $\quad$ Klose, R. J.; Kallin, E. M.; Zhang, Y. Nat. Rev. Genet. 2006, 7, 715.

5. Banerji, B.; Conejo-Garcia, A.; McNeill, L. A.; McDonough, M. A.; Buck, M. R. G.; Hewitson, K. S.; Oldham, N. J.; Schofield, C. J. J. Chem. Soc., Chem. Commun. 2005, 5438. 6. McDonough, M. A.; McNeill, L. A.; Tilliet, M.; Papamicael, C. A.; Chen, Q.-Y.; Banerji, B.; Hewitson, K. S.; Schofield, C. J. J. Am. Chem. Soc. 2005, 127, 7680.

7. McDonough, M. A.; Li, V.; Flashman, E.; Chowdhury, R.; Mohr, C.; Liénard, B. M. R.; Zondlo, J.; Oldham, N. J.; Clifton, I. J.; Lewis, J.; McNeill, L. A.; Kurzeja, R. J. M.; Hewitson, K. S.; Yang, E.; Jordan, S.; Syed, R. S.; Schofield, C. J. Proc. Natl. Acad. Sci. U. S. A. 2006, 103, 9814 .

8. $\quad$ Rose, N. R.; Ng, S. S.; Mecinovic, J.; Lienard, B. M. R.; Bello, S. H.; Sun, Z.; McDonough, M. A.; Oppermann, U.; Schofield, C. J. J. Med. Chem. 2008, 51, 7053.

9. Hirsilä, M.; Koivunen, P.; Günzler, V.; Kivirikko, K. I.; Myllyharju, J. J. Biol. Chem. 2003, $278,30772$.

10. Ivan, M.; Haberberger, T.; Gervasi, D. C.; Michelson, K. S.; Günzler, V.; Kondo, K.; Yang, H.; Sorokina, I.; Conaway, R. C.; Conaway, J. W.; Kaelin, W. G. Proc. Natl. Acad. Sci. U. S. A. 2002, 99, 13459.

11. Majamaa, K.; Hanauske-Abel, H. M.; Günzler, V.; Kivirikko, K. I. Eur. J. Biochem. 1984, 138, 239.

12. Liénard, B. M. R.; Horsfall, L. E.; Galleni, M.; Frère, J.-M.; Schofield, C. J. Bioorg. Med. Chem. Lett. 2007, 17, 964.

13. Thasana, N.; Prachyawarakorn, V.; Tontoolarug, S.; Ruchirawat, S. Tetrahedron Lett. 2003, 44, 1019.

14. $\quad$ Poppe, L.; Tegley, C. M.; Li, V.; Lewis, J.; Zondlo, J.; Yang, E.; Kurzeja, R. J. M.; Syed, R. J. Am. Chem. Soc. 2009, 131, 16654.

15. Elkins, J. M.; Hewitson, K. S.; McNeill, L. A.; Seibel, J. F.; Schlemminger, I.; Pugh, C. W.; Ratcliffe, P. J.; Schofield, C. J. J. Biol. Chem. 2003, 278, 1802.

16. Hewitson, K. S.; Liénard, B. M. R.; McDonough, M. A.; Clifton, I. J.; Butler, D.;

Soares, A. S.; Oldham, N. J.; McNeill, L. A.; Schofield, C. J. J. Biol. Chem. 2007, 282, 3293.

17. Hewitson, K. S.; Holmes, S. L.; Ehrismann, D.; Hardy, A. P.; Chowdhury, R.;

Schofield, C. J.; McDonough, M. A. J. Biol. Chem. 2008, 283, 25971.

18. Rose, N. R.; Woon, E. C. Y.; Kingham, G. L.; King, O. N. F.; Mecinović, J.; Clifton, I. J.; Ng, S. S.; Talib-Hardy, J.; Oppermann, U.; McDonough, M. A.; Schofield, C. J. J. Med. Chem. 2009, 53, 1810.

19. Finnin, M. S.; Donigian, J. R.; Cohen, A.; Richon, V. M.; Rifkind, R. A.; Marks, P. A.; Breslow, R.; Pavletich, N. P. Nature (London) 1999, 401, 188. 